\title{
FGFR1 expression defines clinically distinct subtypes in pancreatic cancer
}

\author{
Farhan Haq ${ }^{1+}$, You-Na Sung ${ }^{2 \dagger}$, Inkeun Park ${ }^{3}$, Mahmood Akhtar Kayani ${ }^{1}$, Faizah Yousuf ${ }^{1}$, Seung-Mo Hong ${ }^{2^{*}}$
} and Sung-Min $\mathrm{Ahn}^{3,4^{*}}$

\begin{abstract}
Background: The clinical significance of fibroblast growth factor receptor 1 (FGFR1) protein expression in pancreatic cancer is largely unknown. In this study, we aimed investigate the clinical significance of FGFR1 expression in pancreatic cancer.

Methods: First, we investigated the relationship between FGFR pathway gene expression and clinicopathological data in three pancreatic cancer cohorts containing 313 cases. Subsequently, to confirm the findings from the discovery cohorts, we performed immunohistochemistry (IHC) of FGFR1 protein in a validation cohort of 205 pancreatic cancer cases.

Results: In discovery cohort 1, FGFR1 and Klotho beta (KLB) overexpression was associated with low tumor stage $(P<0.05)$, low tumor grade $(P<0.05)$, and better overall survival. Multivariate analysis predicted FGFR1 $(P<0.05)$ as a prognostic factor for better overall survival. In discovery cohorts 2 and 3, only FGFR1 overexpression was associated with better overall survival $(P<0.05)$. In the validation cohort, there were $15.7 \%$ and $61 \%$ strong and weak/moderate FGFR1-positive cases, respectively. FGFR1-positive cases showed better overall survival than FGFR1-negative cases $(P<0.05)$. Furthermore, multivariate analysis revealed FGFR1 positivity as an independent prognostic factor for better overall survival in pancreatic cancer patients (hazard ratio $0.677,95 \%$ confidence interval $0.471-0.972, P=0.035$ ).
\end{abstract}

Conclusions: FGFR1 expression, as estimated by IHC, may be used to define clinically distinct subtypes in pancreatic cancer. Moreover, FGFR1-based subclassification of pancreatic cancer may lead to new therapeutic approaches for the FGFR1-positive subtype.

Keywords: FGFR1, Classification, Prognosis, Pancreatic cancer, Anti-FGFR1 therapy

\section{Background}

The fibroblast growth factor receptor (FGFR) pathway is one of the major carcinogenic pathways in cancer [1-5]. Genetic deregulation of fibroblast growth factors and their receptors plays an important role in the initiation and progression of different types of cancer [6-9].

\footnotetext{
*Correspondence: smhong28@gmail.com; smahn@gachon.ac.kr; ahnsungmin@gmail.com

${ }^{\dagger}$ Farhan Haq and You-Na Sung contributed equally to the study

${ }^{2}$ Department of Pathology, Asan Medical Center, University of Ulsan

College of Medicine, Olympic-Ro 43Gil 88, Songpa-Gu, Seoul, Republic of Korea

${ }^{3}$ Division of Oncology, Department of Internal Medicine, Gachon University Gil Hospital, Gachon University Gil Medical Center, Incheon, Republic of Korea

Full list of author information is available at the end of the article
}

Helsten and colleagues [10] reported that the FGFR pathway is the third most frequently altered pathway in cancer, after the p53 and KRAS pathways. Accordingly, cancer drugs targeting the FGFR pathway have been tested in multiple cancers $[1,11]$. Currently, phase I and phase II clinical trials of dovitinib, lucitanib, ponatinib, nintedanib, and pazopanib are underway in different solid tumors to block FGFR pathway activation [12-16].

In pancreatic cancer, aberrations in the FGFR pathway, particularly FGFR1 overexpression, have been reported. According to The Cancer Genome Atlas (TCGA) study on pancreatic cancer [17], FGFR1 is upregulated in approximately $5 \%$ of pancreatic cancers. Lehnen and colleagues [18] reported that FGFR1 was expressed in $4 \%(5 / 125)$ of pancreatic cancer cases, and 
FGFR1 amplification was observed in 2.6\% (4/155). In contrast, Kornmann and colleagues [19] reported that $57 \%(4 / 7)$ of pancreatic cancer cases showed immunoreactivity for the IIIc splice variant of FGFR1 (FGFR1 IIIc). Nevertheless, the clinical significance of FGFR1 protein expression in pancreatic cancer is still largely unknown.

In this study, we aimed to investigate the clinical significance of FGFR1 overexpression in pancreatic cancer. First, we investigated the clinical significance of FGFR pathway genes using the gene expression and clinicopathological data from three pancreatic cancer cohorts containing 313 cases. Then, to confirm the findings from the discovery cohorts, we performed immunohistochemistry (IHC) targeting FGFR1 protein in a validation cohort of 205 pancreatic cancer cases.

\section{Methodology}

\section{Data collection and screening}

The overall study design is described in Additional file 1: Figure S1. Five FGFR pathway genes that are frequently dysregulated in multiple cancers, namely FGFR1, FGFR4, KLB (an FGFR co-receptor), FGF19 (the FGFR4 ligand), and FGF21 (the FGFR1 ligand), were selected for the analysis. Data from discovery cohort 1 , consisting of 65 pancreatic cancer patients, were downloaded from the Gene Expression Omnibus database (Accession \# GSE62452). Clinical features of discovery cohort 1 , including stage, grade, and overall survival information, can be found in Additional file 1: Table S1. LogR expression values of data from the discovery cohort were generated from the Affymetrix Human Gene 1.0 ST array. In discovery cohort 1 , the expression status of FGF19 (probe ID: 7950023), FGF21 (probe ID: 8030105), FGFR1 (probe ID: 8150318), FGFR4 (probe ID: 8110265), and $K L B$ (probe ID: 8094679) were screened for the analysis. In discovery cohort 2 , LogR expression values were generated using the RSTA Custom Affymetrix 2.0 array (Additional file 1: Figure S1). The expression status of FGF19 (probe ID: merck-NM_005117_at), FGF21 (probe ID: merck-NM_019113_at), FGFR1 (probe IDs: merck-NM_000604_at, merck-NM_023110_a_at, and merck2-NM_001174063.1), FGFR4 (probe ID: merckNM_002011_at), and KLB (probe IDs: merck-BC033021_ at and merck-NM_175737_a_at) was analyzed. In discovery cohort 3, RNA-seq data of 179 pancreatic cancers were analyzed (Additional file 1: Figure S1). The expression of FGF19, FGF21, FGFR1, FGFR4, and KLB was estimated using RNA-seq data with a $\mathrm{z}$-score $>2.0$. All detailed information from the pancreatic cancer dataset is available in the public cBioPortal database (Pancreatic Adenocarcinoma, TCGA, provisional).

\section{Statistical analysis}

The associations of FGF19, FGF21, FGFR1, FGFR4, and $K L B$ expression with clinical features, including stage, grade, and survival, were calculated using $\chi^{2}$ and Fisher exact tests for the three discovery cohorts. Survival analysis was performed using Kaplan-Meier curves with logrank (Mantel-Cox) P values. Cox proportional hazard regression and univariate and multivariable analyses were used to evaluate the association between gene expression and survival. Since the sample sizes of the discovery cohorts were small, the multivariable Cox regression model may have led to the overfitting of the data. Therefore, each gene was analyzed separately in combination with the clinical features in multivariable analysis. The hazard ratio (HR) and 95\% confidence interval (CI) were also calculated for each factor. $\mathrm{P}$ values were two-sided, and $P<0.05$ was considered to be statistically significant. All statistical analyses were performed with SPSS 21.0 software (IBM, Armonk, NY, USA).

\section{Validation in $\mathbf{2 0 5}$ patients using IHC}

Immunohistochemical labeling was performed in a validation cohort of 205 pancreatic cancer patients at the immunohistochemical laboratory of the Department of Pathology, Asan Medical Center. In brief, 4- $\mu \mathrm{m}$-thick sections were deparaffinized with xylenes and hydrated in an ethanol series. Endogenous peroxidase activity was blocked by incubation in $3 \% \mathrm{H}_{2} \mathrm{O}_{2}$ for $10 \mathrm{~min}$, and then heat-induced antigen retrieval was performed. Primary antibodies were used with a Benchmark autostainer (Ventana Medical Systems, Tucson, AZ, USA) in accordance with the manufacturer's protocol. Sections were incubated at room temperature for 32 min in primary antibody for FGFR1 (rabbit polyclonal, 1:100; Abnova, Taipei, Taiwan). The sections were then labeled with an automated immunostaining system and processed with an iView DAB detection kit (Benchmark XT, Ventana Medical Systems). Immunostained sections were lightly counterstained with hematoxylin, dehydrated in ethanol, and cleared in xylenes. Immunoreactivity was interpreted by light microscopic examination and independently evaluated by two pathologists, coauthors of this study (Y.N.S. and S.M.H.), who were blind to the clinicopathologic information. Cases were categorized as positive, weak positive and negative.

\section{Results \\ Expression analysis of FGFR genes in discovery cohort 1}

The $\log \mathrm{R}$ expression values of five FGFR-related genes were dichotomized according to their median expressions (Additional file 1: Figure S2). FGFR1 had the 
highest expression, with a median enrichment of 5.922, whereas $F G F 21$ had the lowest expression, with a median enrichment of 2.884 . The expression level of FGFR4 was significantly correlated with those of FGF19 (Pearson Correlation $=0.30, P=0.014$ ), KLB (Pearson Correlation $=0.41, P=0.001$ ), and FGFR1 (Pearson Correlation $=0.29, P=0.02)$. Notably, a strong correlation was observed between FGFR1 and KLB expression (Pearson's correlation $=0.60, P<0.001$ ). FGF21 expression was not correlated with that of any of the other four genes.

\section{Association between FGFR genes and clinical features in discovery cohort 1}

According to Fisher's exact test results, overexpression of FGFR4 $(P<0.001)$ and $K L B(P=0.005)$ were significantly associated with a low tumor grade. Overexpression of FGFR1 was significantly associated with both low tumor grade $(P=0.023)$ and low tumor stage $(P=0.023)$. FGF19 and FGF21 overexpression did not show any association with clinical features.

Next, the association between FGF19, FGF21, FGFR1, FGFR4, and KLB overexpression and the overall survival of pancreatic cancer patients was evaluated. According to univariate analysis, FGFR1 overexpression (HR 0.475, 95\% CI 0.277-0.817, $P=0.007$ ) and $K L B$ overexpression (HR $0.536,95 \%$ CI $0.318-0.903$, $P=0.019)$ were significantly associated with better overall survival. In addition, FGFR4 overexpression showed a trend toward better overall survival, but it did not reach statistical significance (HR 0.610, 95\% CI $0.370-1.008, P=0.054$ ) (Table 1). Kaplan-Meier curves also showed a clear separation between patients with high and low expression of FGFR1 (Fig. 1a), KLB (Fig. 1b), and FGFR4 (Fig. 1c). Furthermore, multivariable analysis was performed along with the prognostic factors (tumor stage and tumor grade) associated with pancreatic cancer. FGFR1 expression was the only prognostic factor for better overall survival $(\mathrm{HR}=0.524$, 95\% CI 0.281-0.977, $P=0.042$ ) (Table 2).

\begin{tabular}{lcc}
$\begin{array}{l}\text { Table } \mathbf{1} \text { Univariate } \\
\text { for overall survival in cohort } \mathbf{1}\end{array}$ & genes \\
\hline Gene name & Hazard ratio $(\mathbf{9 5} \% \mathbf{C l})$ & P value \\
\hline FGFR1 & $0.475(0.277-0.817)$ & 0.007 \\
FGFR4 & $0.610(0.370-1.008)$ & 0.054 \\
FGF19 & $1.029(0.630-1.682)$ & 0.908 \\
FGF21 & $0.853(0.515-1.413)$ & 0.537 \\
KLB & $0.536(0.318-0.903)$ & 0.019 \\
\hline
\end{tabular}

\section{Expression and survival analysis of FGFR genes}

\section{in discovery cohort 2}

The potential significance of the FGFR-pathway genes was further evaluated in cohort 2. Similarly, $\log R$ expression values of the five FGFR-related genes were dichotomized according to their medians (Additional file 1: Figure S3). Consistent with the results in cohort 1, the highest median expression was observed for FGFR1. Of note, of all five genes, only patients with FGFR1 expression had significantly better overall survival by KaplanMeier analysis (Fig. 1d).

\section{Expression and survival analysis of FGFR genes in discovery cohort 3}

The TCGA pancreatic cancer dataset, which includes data from 179 pancreatic cancer patients, was selected as cohort 3. Overexpression of FGFR1, KLB, FGF19, FGFR4, and FGF21 was identified in 7 (4\%), 7 (4\%), 6 (3\%), $5(2.8 \%)$, and $2(1.1 \%)$ patients, respectively (Additional file 1: Figure S4A). Notably, only FGFR1 overexpression was significantly associated with better overall $(P=0.0158)$ and disease-free survival $(P=0.006)$ (Additional file 1: Figure S4B).

\section{FGFR1 immunolabeling in the validation cohort}

Representative IHC images of FGFR1-positive and -negative cases from the validation cohort are depicted in Fig. 2a, b, respectively. Thirty (15.7\%) cases were strongly FGFR1-positive, and 118 (61\%) were moderately/weakly positive. Forty-three cases (22.5\%) were negative for FGFR1 expression.

\section{Univariate and multivariate analysis for overall survival}

According to the statistical analysis, no significant associations were observed between FGFR1 positivity and poor clinicopathological features, including bile duct invasion, duodenal invasion, perineural invasion, lymphovascular invasion, and lymph node metastasis. However, in univariate analysis, overall survival was significantly associated with age (HR 1.451, 95\% CI 1.073-1.961, $P=0.016$ ), bile duct invasion (HR 1.469, 95\% CI 1.099-1.963, $P=0.009$ ), lymphovascular invasion (HR 1.455, 95\% CI 1.088-1.944, $P=0.011$ ), lymph node metastasis (HR 2.495, 95\% CI 1.811-3.436, $P<0.001$ ), and FGFR1 expression (HR $0.590,95 \%$ CI $0.415-0.839, P=0.003$ ) (Table 3). Furthermore, in the multivariate analysis, age $(\mathrm{HR}=1.587,95 \%$ CI 1.130-2.230, $P=0.008$ ), bile duct invasion (HR 1.657, 95\% CI 1.211-2.269, $P=0.002)$, lymph node metastasis (HR 2.360, 95\% CI 1.665-3.344, $P<0.001$ ), and FGFR1 expression (HR 0.677, 95\% CI 0.471-0.972, $P=0.035$ ) were predicted as significant prognostic biomarkers for 

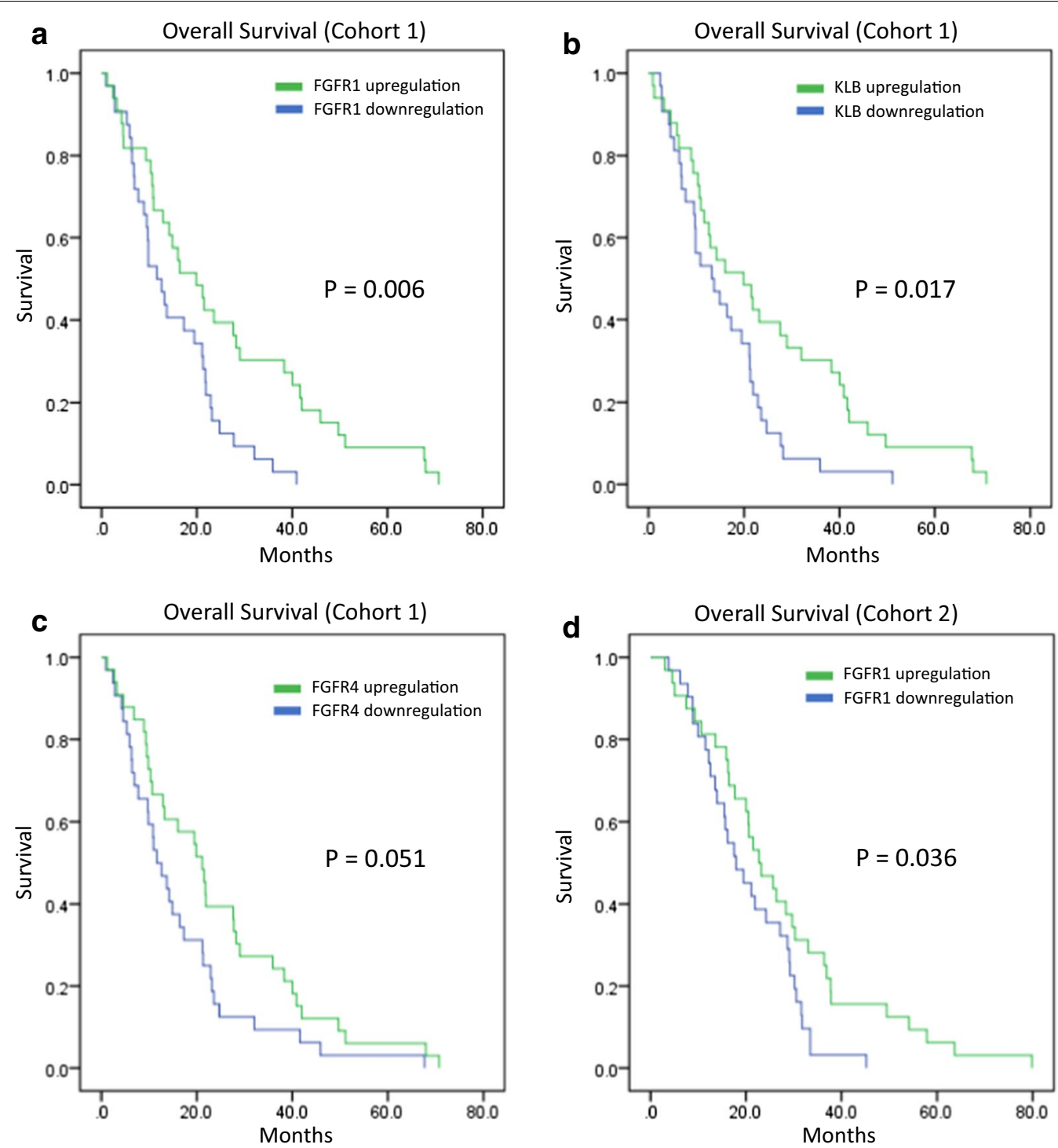

Fig. 1 Kaplan-Meier survival analyses of FGFR1, FGFR4, and KLB in pancreatic cancer cohorts 1 and 2. Green represents expression $\geq$ the median, while blue represents expression < the median. Overall survival by expression of FGFR1 (a), KLB (b), and FGFR4 (c) in cohort 1. Overall survival by expression of FGFR1 (d) in cohort 2. FGFR fibroblast growth factor receptor, KLB klotho beta

overall survival in pancreatic cancer. Notably, FGFR1 expression was the only prognostic biomarker for better overall survival (i.e., $\mathrm{HR}<1$ ) (Table 3).

\section{Discussion}

The main finding of this study is that FGFR1 protein expression defines clinically distinct subtypes of pancreatic cancer. FGFR1-positive cases showed better overall survival than FGFR1-negative cases. To the best of our knowledge, this study is the first to demonstrate the clinical significance of FGFR1 overexpression in pancreatic cancer.
The clinical significance of FGFR1 overexpression has not been thoroughly investigated in pancreatic cancer for two reasons: (1) KRAS mutation, which does not have any targeted solution, is almost universal in pancreatic cancer; (2) FGFR1 overexpression is not exclusive with KRAS mutation $[17,20]$. Although, recent genomic and transcriptomic studies identified new subtypes of PADC, but prognostic role of FGFR1 is not highlighted. For instance, Bailey and colleagues demonstrated that $\mathrm{Kras}^{\mathrm{G12D/}} ; \mathrm{Tr}$ $p 53^{f l+}$ mutant subtype (containing group of genes with FGFR1 expression) showed less aggressive clinical outcome compared to $\mathrm{Kras}{ }^{G 12 \mathrm{D} /+} ; \operatorname{Trp} 53^{f l /+} ; \mathrm{TAp} 3^{f / f l}$ mutant 
Table 2 Multivariate analysis of FGFR-related genes for overall survival in cohort 1

\begin{tabular}{lll}
\hline Factors & Hazard ratio $\mathbf{9 5 \%}$ Cl) & P value \\
\hline FGFR1 & & \\
FGFR1 (high vs. low) & $0.524(0.281-0.977)$ & 0.042 \\
Tumor grade $(1,2$ vs. 3, 4) & $0.585(0.337-1.016)$ & 0.057 \\
Tumor stage $(1,2$ vs. 3, 4) & $0.814(0.427-1.548)$ & 0.530 \\
FGFR4 & & \\
FGFR4 (high vs. low) & $0.975(0.516-1.842)$ & 0.937 \\
Tumor grade $(1,2$ vs. 3, 4) & $0.514(0.263-1.002)$ & 0.051 \\
Tumor stage $(1,2$ vs. 3, 4) & $1.109(0.614-2.001)$ & 0.732 \\
KLB & & \\
KLB (high vs. low) & $0.640(0.375-1.093)$ & 0.102 \\
Tumor grade $(1,2$ vs. 3, 4) & $0.547(0.316-0.945)$ & 0.031 \\
Tumor stage (1, 2 vs. 3, 4) & $1.082(0.606-1.931)$ & 0.791 \\
\hline
\end{tabular}

subtype (containing group of genes without FGFR1 expression) in pancreatic cancer [21]. Furthermore, another transcriptomic profiling study classified PADC patients into L1-L6 subtypes. Interestingly, the L5 subtype which showed most favorable clinical outcome from the rest of the molecular subtypes was also enriched with FGFR1 pathway related genes [22].

Our finding can have immediate clinical ramifications. In pancreatic cancer, FGFR1 is the most frequently altered receptor of the four FGFR receptors, and, using readily applicable immunohistochemistry methods, FGFR1 can be used to classify pancreatic cancer into FGFR1-positive and -negative subtypes. As FGFR1-positive pancreatic cancer has better prognosis, FGFR1 can be used as an independent predictor of better overall survival in pancreatic cancer patients. Moreover, FGFR1based subclassification of pancreatic cancer may lead to new therapeutic approaches for the FGFR1-positive subtype. For example, it may be possible to target FGFR1 using antibody in strongly FGFR1-positive pancreatic cancer, in the same manner in which HER2 is targeted using anti-HER2 antibodies in HER2-positive breast cancer.

Only a few previous studies have examined FGFR1 expression in pancreas cancers, and they reported a wide range of FGFR1 positivity (4-57\%) [18, 19, 23]. Lehnen and colleagues observed that $4 \%(5 / 125)$ of pancreatic cancer patients showed FGFR1 expression, and amplification was noted in $2.6 \%$ of the cases (4/155) [18]. In contrast, Kornmann et al. reported that $57 \%$ (4/7) of pancreas cancers showed immunoreactivity for FGFR1 IIIc [19]. Our study is unique in that we evaluated FGFR1 expression based on the intensity of FGFR1 labeling, and we observed strong FGFR1 positivity in $15.7 \%$ (30 cases) and moderate/weak FGFR1 positivity in 61.8\% (118 cases) in a cohort of Korean pancreatic cancer patients.
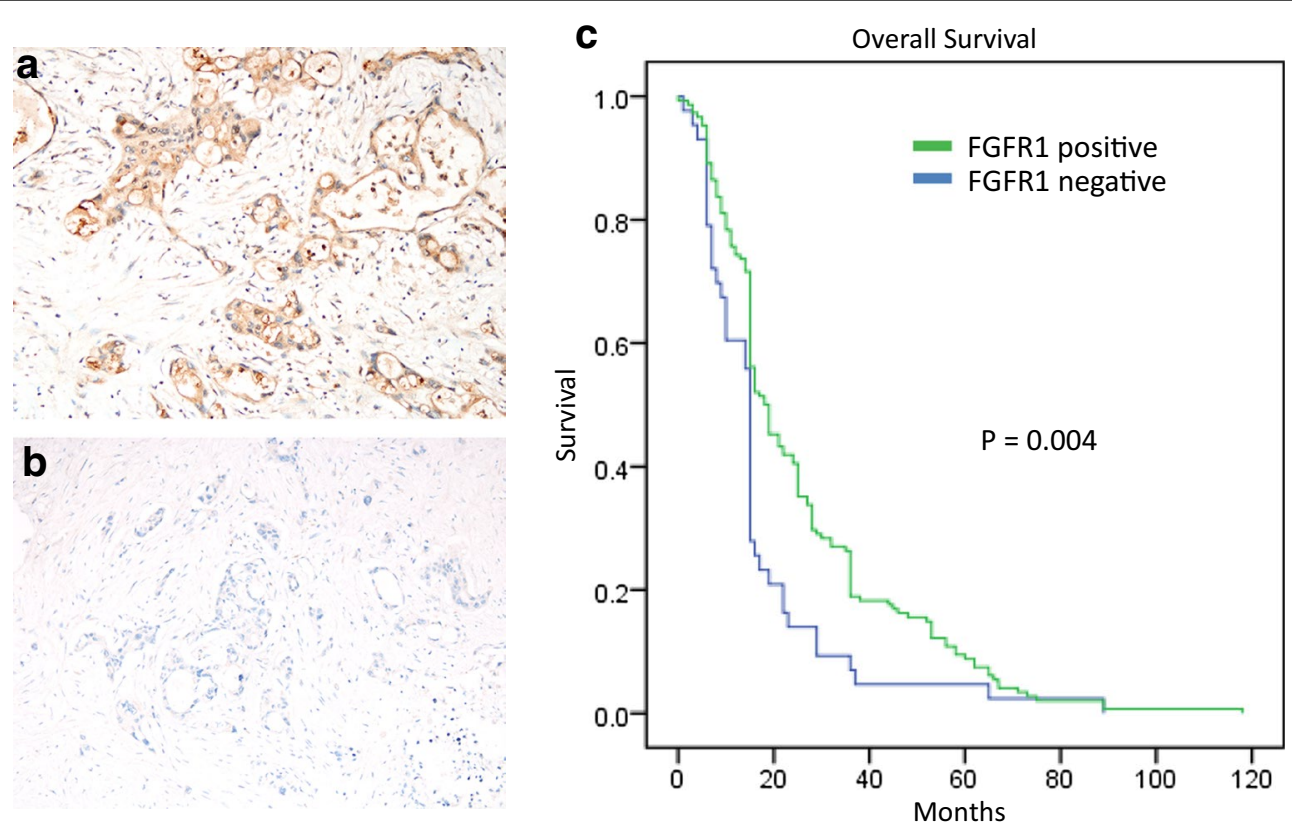

Fig. 2 Immunohistochemistry and Kaplan-Meier survival analysis of FGFR1 in the validation cohort of 205 pancreatic cancer patients. a, b Represent immunohistochemistry results of FGFR1-positive and-negative cases, respectively. c Overall survival of pancreatic cancer patients in the discovery cohort with high and low FGFR1 (c). FGFR: fibroblast growth factor receptor 
Table 3 Cox regression analysis for overall survival in 205 pancreatic cancer patients

\begin{tabular}{|c|c|c|c|c|c|c|c|c|}
\hline & \multirow[t]{3}{*}{$P$ value } & \multirow[t]{3}{*}{ HR } & \multirow{2}{*}{\multicolumn{2}{|c|}{$\begin{array}{l}\text { Univariate Analysis } \\
95.0 \% \mathrm{Cl} \text { for HR }\end{array}$}} & \multirow[t]{3}{*}{$P$ value } & \multirow[t]{3}{*}{ HR } & \multirow{2}{*}{\multicolumn{2}{|c|}{$\begin{array}{l}\begin{array}{l}\text { Multivariate } \\
\text { analysis }\end{array} \\
95.0 \% \mathrm{Cl} \text { for } \mathrm{HR}\end{array}$}} \\
\hline & & & & & & & & \\
\hline & & & Lower & Upper & & & Lower & upper \\
\hline Age, years ( $\geq 60$ vs. $<60)$ & 0.016 & 1.451 & 1.073 & 1.961 & \multirow[t]{5}{*}{0.008} & \multirow[t]{5}{*}{1.587} & \multirow[t]{5}{*}{1.130} & \multirow[t]{5}{*}{2.230} \\
\hline Gender & 0.235 & 1.191 & 0.892 & 1.591 & & & & \\
\hline Tumor size, $\mathrm{cm}(\geq 2$ vs. $<2$ ) & 0.241 & 1.534 & 0.751 & 3.135 & & & & \\
\hline Differentiation (well/moderate vs. poor) & 0.076 & 1.428 & 0.964 & 2.115 & & & & \\
\hline Extension (confined vs. peripancreatic soft tissue) & 0.061 & 1.898 & 0.970 & 3.714 & & & & \\
\hline Bile duct invasion (absent vs. present) & 0.009 & 1.469 & 1.099 & 1.963 & \multirow[t]{2}{*}{0.002} & \multirow[t]{2}{*}{1.657} & \multirow[t]{2}{*}{1.211} & \multirow[t]{2}{*}{2.269} \\
\hline Duodenum invasion (absent vs. present) & 0.074 & 1.303 & 0.975 & 1.742 & & & & \\
\hline Lymphovascular invasion (absent vs. present) & 0.011 & 1.455 & 1.088 & 1.944 & \multirow[t]{2}{*}{0.098} & \multirow[t]{2}{*}{1.320} & \multirow[t]{2}{*}{.950} & \multirow[t]{2}{*}{1.834} \\
\hline Perineural invasion (absent vs. present) & 0.086 & 1.403 & .953 & 2.064 & & & & \\
\hline Lymph node metastasis (absent vs. present) & $<0.001$ & 2.495 & 1.811 & 3.436 & \multirow[t]{2}{*}{$<0.0001$} & \multirow[t]{2}{*}{2.360} & \multirow[t]{2}{*}{1.665} & \multirow[t]{2}{*}{3.344} \\
\hline Tumor stage ${ }^{a}$ & 0.059 & 1.382 & 0.988 & 1.933 & & & & \\
\hline FGFR1 (positive vs. negative) & 0.003 & 0.590 & 0.415 & 0.839 & 0.035 & 0.677 & 0.471 & 0.972 \\
\hline
\end{tabular}

a Tumor stage was not included for multivariate analysis because tumor size, in addition to bile duct and duodenal invasion and extension (confined vs peripancreatic soft tissue extension), are components of T stage

Using only the strong FGFR1 expression group, the frequency of FGFR1 labeling was higher than the results of Lehnen and colleagues' study. However, if we include moderate/weak FGFR1 labeling, the frequency was similar to that of Kornmann's study. This wide range of FGFR1 labeling results may be plausibly explained by different FGFR1 expression rates based on different ethnic groups (Korean and Western populations), use of different antibody clones, or different cutoffs for FGFR1 labeling.

\section{Conclusion}

In summary, FGFR1 overexpression, evaluated by IHC, may be used as a prognostic biomarker for overall survival in pancreatic cancer patients. Moreover, FGFR1 overexpression may define a subset of pancreatic cancer, leading to new therapeutic approaches.

\section{Additional file}

Additional file 1. Additional figures and table.

\section{Authors' contributions}

FH, YNS, MAK and FY carried out experiments and analyzed the data. YNS and IP carried out statistical analyses. SMA and SMH contributed to the conception and design of the study. FH, SMA and SMH contributed to the writing of the manuscript. All authors read and approved the final manuscript.

\section{Author details}

1 Department of Biosciences, COMSATS University, Islamabad, Pakistan.

2 Department of Pathology, Asan Medical Center, University of Ulsan College of Medicine, Olympic-Ro 43Gil 88, Songpa-Gu, Seoul, Republic of Korea.
${ }^{3}$ Division of Oncology, Department of Internal Medicine, Gachon University Gil Hospital, Gachon University Gil Medical Center, Incheon, Republic of Korea. ${ }^{4}$ Department of Genome Medicine and Science, College of Medicine, Gachon Institute of Genome Medicine and Science, Gachon University, Seongnam, Republic of Korea.

\section{Acknowledgements}

Not applicable.

\section{Competing interests}

The authors declare that they have no competing interests.

Availability of data and materials

The links of data are mentioned in "Methodology" section.

\section{Consent for publication}

Not applicable.

\section{Ethics approval and consent to participate}

Not applicable.

\section{Funding}

This research was supported by a grant from the Korea Health Technology R\&D Project through the Korea Health Industry Development Institute, funded by the Ministry of Health and Welfare (HI16C1985) and by a grant from Gachon University Gil Medical Center (FRD2016-20).

\section{Publisher's Note}

Springer Nature remains neutral with regard to jurisdictional claims in published maps and institutional affiliations.

Received: 21 September 2018 Accepted: 12 December 2018

Published online: 28 December 2018

\section{References}

1. Touat M, lleana E, Postel-Vinay S, André F, Soria J-C. Targeting FGFR signaling in cancer. Clin Cancer Res. 2015;21:2684-94.

2. Ho HK, Németh G, Ng YR, Pang E, Szántai-Kis C, Zsákai L, et al. Developing FGFR4 inhibitors as potential anti-cancer agents via in silico 
design, supported by in vitro and cell-based testing. Curr Med Chem. 2013;20:1203-17.

3. Leung HY, Gullick WJ, Lemoine NR. Expression and functional activity of fibroblast growth factors and their receptors in human pancreatic cancer. Int J Cancer. 1994;59:667-75.

4. Yoo C, Kang J, Kim D, Kim K-P, Ryoo B-Y, Hong S-M, et al. Multiplexed gene expression profiling identifies the FGFR4 pathway as a novel biomarker in intrahepatic cholangiocarcinoma. Oncotarget. 2017;8:38592-601.

5. Ahn S-M, Jang SJ, Shim JH, Kim D, Hong S-M, Sung CO, et al. Genomic portrait of resectable hepatocellular carcinomas: implications of RB1 and FGF19 aberrations for patient stratification. Hepatology. 2014;60:1972-82.

6. Turner N, Pearson A, Sharpe R, Lambros M, Geyer F, Lopez-Garcia MA, et al. FGFR1 amplification drives endocrine therapy resistance and is a therapeutic target in breast cancer. Cancer Res. 2010;70:2085-94.

7. Miura S, Mitsuhashi N, Shimizu H, Kimura F, Yoshidome H, Otsuka M, et al. Fibroblast growth factor 19 expression correlates with tumor progression and poorer prognosis of hepatocellular carcinoma. BMC Cancer. 2012;12:56.

8. Fumarola C, Cretella D, Monica SL, Bonelli MA, Alfieri R, Caffarra C, et al. Enhancement of the anti-tumor activity of FGFR1 inhibition in squamous cell lung cancer by targeting downstream signaling involved in glucose metabolism. Oncotarget. 2017;8:91841-59.

9. Turner N, Grose R. Fibroblast growth factor signalling: from development to cancer. Nat Rev Cancer. 2010;10:116-29.

10. Helsten T, Elkin S, Arthur E, Tomson BN, Carter J, Kurzrock R. The FGFR landscape in cancer: analysis of 4,853 tumors by next-generation sequencing. Clin Cancer Res Off J Am Assoc Cancer Res. 2016;22:259-67.

11. Babina IS, Turner NC. Advances and challenges in targeting FGFR signalling in cancer. Nat Rev Cancer. 2017;17:318-32.

12. Porta C, Giglione P, Liguigli W, Paglino C. Dovitinib (CHIR258, TKI258): structure, development and preclinical and clinical activity. Future Oncol Lond Engl. 2015;11:39-50.

13. Chae YK, Ranganath K, Hammerman PS, Vaklavas C, Mohindra N, Kalyan A, et al. Inhibition of the fibroblast growth factor receptor (FGFR) pathway: the current landscape and barriers to clinical application. Oncotarget. 2016;8:16052-74.

14. Gozgit JM, Wong MJ, Moran L, Wardwell S, Mohemmad QK, Narasimhan $\mathrm{NI}$, et al. Ponatinib (AP24534), a multitargeted Pan-FGFR inhibitor with activity in multiple FGFR-amplified or mutated cancer models. Mol Cancer Ther. 2012;11:690-9.

15. Dhillon S. Nintedanib: a review of its use as second-line treatment in adults with advanced non-small cell lung cancer of adenocarcinoma histology. Target Oncol. 2015;10:303-10.

16. Hamberg P, Verweij J, Sleijfer S. (Pre-)clinical pharmacology and activity of pazopanib, a novel multikinase angiogenesis inhibitor. Oncologist. 2010;15:539-47.

17. Cancer Genome Atlas Research Network. Electronic address: andrew aguirre@dfci.harvard.edu, Cancer Genome Atlas Research Network. Integrated genomic characterization of pancreatic ductal adenocarcinoma. Cancer Cell. 2017;32:185-203.e13.

18. Lehnen NC, von Mässenhausen $A$, Kalthoff $H$, Zhou H, Glowka T, Schütte $\mathrm{U}$, et al. Fibroblast growth factor receptor 1 gene amplification in pancreatic ductal adenocarcinoma. Histopathology. 2013;63:157-66.

19. Kornmann M, Ishiwata T, Matsuda K, Lopez ME, Fukahi K, Asano G, et al. IIIc isoform of fibroblast growth factor receptor 1 is overexpressed in human pancreatic cancer and enhances tumorigenicity of hamster ductal cells. Gastroenterology. 2002;123:301-13.

20. Zeitouni D, Pylayeva-Gupta Y, Der CJ, Bryant KL. KRAS mutant pancreatic cancer: no lone path to an effective treatment. Cancers (Basel). 2016;8:E45

21. Bailey P, Chang DK, Nones K, Johns AL, Patch A-M, Gingras M-C, et al. Genomic analyses identify molecular subtypes of pancreatic cancer. Nature. 2016;531:47-52.

22. Zhao L, Zhao H, Yan H. Gene expression profiling of 1200 pancreatic ductal adenocarcinoma reveals novel subtypes. BMC Cancer. 2018;18:603.

23. Kobrin MS, Yamanaka Y, Friess H, Lopez ME, Korc M. Aberrant expression of type I fibroblast growth factor receptor in human pancreatic adenocarcinomas. Cancer Res. 1993;53:4741-4.
Ready to submit your research? Choose BMC and benefit from:

- fast, convenient online submission

- thorough peer review by experienced researchers in your field

- rapid publication on acceptance

- support for research data, including large and complex data types

- gold Open Access which fosters wider collaboration and increased citations

- maximum visibility for your research: over $100 \mathrm{M}$ website views per year

At $\mathrm{BMC}$, research is always in progress.

Learn more biomedcentral.com/submissions 